\title{
Gadolinium Enhancement in a Case of Uncomplicated Posterior Reversible Encephalopathy Syndrome
}

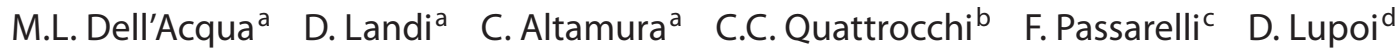 \\ P.M. Rossini ${ }^{a}, c, e \quad$ F. Vernieri ${ }^{a}$ e \\ Departments of ${ }^{a}$ Neuroscience and ${ }^{b}$ Radiology, Università Campus Bio-Medico, Departments of ${ }^{c}$ Neuroscience \\ and ${ }^{\mathrm{d}}$ Radiology, Ospedale Fatebenefratelli, and ${ }^{\mathrm{e}}$ Associazione Fatebenefratelli per la Ricerca, Rome, Italy
}

\begin{abstract}
Dear Sir,
Posterior reversible encephalopathy syndrome (PRES) is typically related to severe high systemic blood pressure (SBP) but may occur with a mildly elevated or normal SBP. MRI characteristic findings are hyperintense areas on $\mathrm{T}_{2}$-weighted images, usually resolving if the underlying causes are promptly treated [1]. Early differentiation between reversible and permanent lesions is not possible with conventional MRI. Gadolinium contrast enhancement is not considered a feature of PRES and, if present, is considered a marker of adverse outcome.
\end{abstract}

\section{Case Report}

A 29-year-old, previously healthy, primiparous woman in the 28th week of gestation was admitted to the Obstetrics Unit for a pre-eclamptic syndrome and treated with antihypertensive drugs. Laboratory findings were normal. Foetal ultrasound showed intra-uterine growth restriction leading to caesarean section. The day after delivery, her SBP was high in spite of therapy. Suddenly, she complained of blindness. Neurological examination was normal except for visual perception: she could not recognize objects, although she was able to follow them in the visual field. Ce- rebral CT was normal. Serum lactic dehydrogenase (LDH) levels rapidly increased. After $2 \mathrm{~h}$ she presented a right-sided motor seizure. Few hours later she was no longer able to follow objects in the visual field. Neurological examination was negative for other focal signs.

About $10 \mathrm{~h}$ after the clinical onset, the patient underwent an MRI examination that showed hyperintense areas on fluidattenuated inversion recovery (FLAIR; fig. $1 \mathrm{~B}, \mathrm{H}$ ) and fast spin echo $\mathrm{T}_{2}$-weighted sequences, in the occipital cortex and in the subcortical white matter bilaterally, more evident in the deep white matter of the left frontoparietal lobe. Diffusion-weighted images showed restricted diffusion of the left frontal lesion (fig. 1A). In the same location and in the occipital lobe bilaterally (fig. 1C, I), contrast-enhanced images showed blood-brain barrier (BBB) disruption. After few hours, SBP normalized. The LDH serum level decreased gradually. The cortical blindness recovered within $24 \mathrm{~h}$ and no other seizures occurred.

Magnetic resonance studies conducted 15 days and 6 weeks later detected only a minimal hyperintensity on FLAIR images with a dramatic volume reduction of the left frontal lesion (fig. 1E), corresponding to the one with previous diffusion restriction and gadolinium enhancement.

\section{Discussion}

Controversial observations are reported about post-gadolinium magnetic resonance images in PRES. When MRI was performed 6-48 h after onset, no patients presented contrast-enhancing areas in the absence of ischaemic complications, suggesting that BBB leakage is not typical of PRES [2]. Conversely, anecdotal uncomplicated cases presented gadolinium-en-

Fig. 1. MRI examination $10 \mathrm{~h}$ after the clinical onset (A-C and $\mathbf{G}-\mathbf{I}$ ) and 15 days later (D-F and $\mathbf{J}-\mathbf{L})$. Diffusion-weighted sequences $(\mathbf{A}, \mathbf{D}, \mathbf{G}, \mathbf{J})$ show restricted diffusion in the left frontal lesion, only at clinical onset. FLAIR images $(\mathbf{B}, \mathbf{E}, \mathbf{H}, \mathbf{K})$ show bilateral, frontal and occipital lobe hyperintensities at clinical onset, and slight hyperintensity after 15 days, only in the left frontal lesion. Contrast-enhanced $\mathrm{T}_{1}$-weighted images $(\mathbf{C}, \mathbf{F}, \mathbf{I}, \mathbf{L})$ show $\mathrm{BBB}$ disruption of the left frontal and occipital lesions, only at clinical onset. After 15 days, only a slight hyperintensity persists at the level of the left frontal lesion (E), whereas occipital hyperintensities are no more detectable (K).

\section{KARGER}

Fax +41 613061234

E-Mail karger@karger.ch

www.karger.com (c) 2008 S. Karger AG, Basel

0014-3022/08/0594-0208\$24.50/0

Accessible online at:

www.karger.com/ene
Dr. F. Vernieri, MD

Università Campus Bio-Medico di Roma

Via dei Compositori, 130

IT-00128 Rome (Italy)

Tel. +39 0622541 645, Fax +39 0622541 6879, E-Mail f.vernieri@unicampus.it 

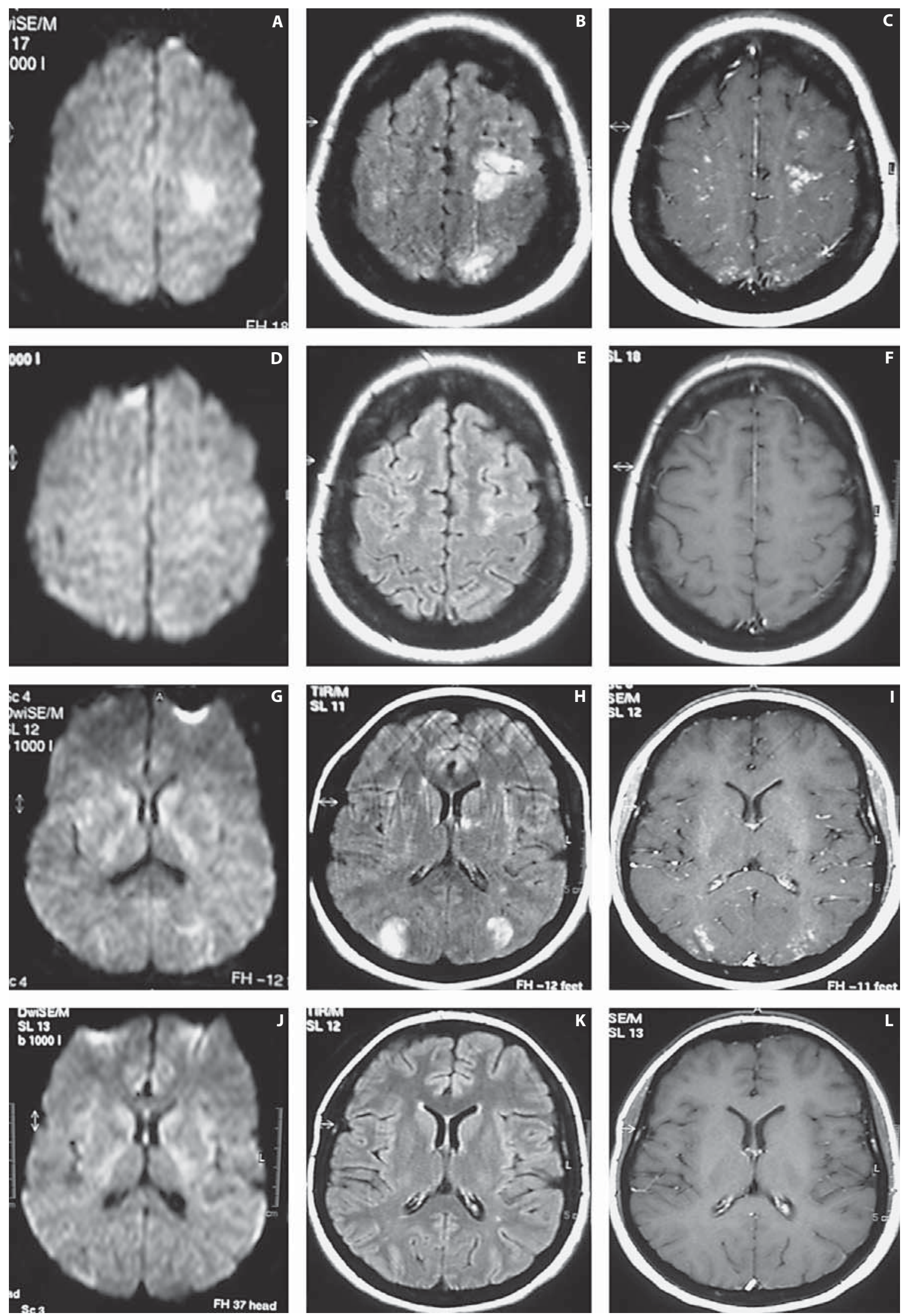

Gadolinium Enhancement in a Case of

Eur Neurol 2008:59:208-210 
hancing lesions, when MRI was performed around $5 \mathrm{~h}$ from clinical onset. This finding supports the hypothesis of a transient disruption of the $\mathrm{BBB}$, which resolves within few hours.

In our case, a 10-hour-lasting BBB disruption, not associated with permanent brain damage, could be explained by a mechanism previously hypothesized for transient ischaemia. In fact, after acute transient ischaemia the $\mathrm{BBB}$ opens immediately, leading to gadolinium (molecular weight $938 \mathrm{Da}$ ) extravasation, and closes within minutes to hours, blocking the diffusion of substances heavier than $600 \mathrm{Da}$ but allowing the development of vasogenic oedema [3].

An altered autoregulation usually subtends PRES. It physiologically preserves a constant blood flow through myogenic and neurogenic mechanisms. When the myogenic mechanism fails, autoregulation depends only on the sympathetic innervation of the adventitia. Thus, excessive SBP or endothelial dysfunction promotes breakthrough of autoregulation where sympathetic innervation is scanty as in the posterior circulation [4].

A preserved endothelial function is necessary to detect and respond to SBP changes. Several factors, such as immunosuppressive or cytotoxic drugs, trigger PRES probably by a toxic effect on vascular endothelium even in normotensive patients. In eclampsia, the secretion of trophoblastic cytotoxic factors sustains the maternal endothelial dysfunction. Laboratory findings such as an $\mathrm{LDH}$ increase support the pathogenic role of endothelial dysfunction in PRES.

On this basis we can argue that, in our patient, the BBB permeability to gadolini- um in the absence of ischaemic injury may be a marker of prolonged endothelial dysfunction, not associated with adverse outcome.

\section{References}

1 Hinchey J, Chaves C, Appignani B, et al: A reversible posterior leukoencephalopathy syndrome. N Engl J Med 1996;334:494-500.

2 Ugurel MS, Hayakawa M: Implication of post-gadolinium MRI results in 13 cases with posterior reversible encephalopathy syndrome. Eur J Radiol 2005;53:441-449.

3 Lo EH, Singhal AB, Torchilin VP, Abbott NJ: Drug delivery to damaged brain. Brain Res Brain Res Rev 2001;38:140-148.

4 Van Mook WN, Rennenberg RJ, Schurink $\mathrm{GW}$, et al: Cerebral hyperperfusion syndrome. Lancet Neurol 2005;4:877-888. 